\title{
Production and Decay of Element 114: High Cross Sections and the New Nucleus ${ }^{277} \mathrm{Hs}$
}

\author{
Ch. E. Düllmann, ${ }^{1, *}$ M. Schädel, ${ }^{1}$ A. Yakushev, ${ }^{2}$ A. Türler, ${ }^{2, \dagger}$ K. Eberhardt, ${ }^{3}$ J. V. Kratz, ${ }^{3}$ D. Ackermann, ${ }^{1}$
} L.-L. Andersson, ${ }^{4}$ M. Block, ${ }^{1}$ W. Brüchle, ${ }^{1}$ J. Dvorak, ${ }^{5,6}$ H. G. Essel, ${ }^{1}$ P. A. Ellison, ${ }^{5,6}$ J. Even, ${ }^{3}$ J. M. Gates, ${ }^{1,2}$ A. Gorshkov, ${ }^{2}$ R. Graeger, ${ }^{2}$ K. E. Gregorich, ${ }^{5}$ W. Hartmann, ${ }^{1}$ R.-D. Herzberg, ${ }^{4}$ F. P. Heßberger, ${ }^{1}$ D. Hild, ${ }^{3}$ A. Hübner, ${ }^{1}$ E. Jäger, ${ }^{1}$ J. Khuyagbaatar, ${ }^{1}$ B. Kindler, ${ }^{1}$ J. Krier, ${ }^{1}$ N. Kurz, ${ }^{1}$ S. Lahiri, ${ }^{7}$ D. Liebe, ${ }^{3}$ B. Lommel,,${ }^{1}$ M. Maiti, ${ }^{7}$ H. Nitsche,${ }^{5,6}$ J. P. Omtvedt, ${ }^{8}$ E. Parr, ${ }^{4}$ D. Rudolph, ${ }^{9}$ J. Runke, ${ }^{3}$ B. Schausten, ${ }^{1}$ E. Schimpf,${ }^{1}$ A. Semchenkov, ${ }^{8}$ J. Steiner, ${ }^{1}$ P. Thörle-Pospiech, ${ }^{3}$ J. Uusitalo, ${ }^{10}$ M. Wegrzecki, ${ }^{11}$ and N. Wiehl ${ }^{3}$

${ }^{1}$ GSI Helmholtzzentrum für Schwerionenforschung GmbH, 64291 Darmstadt, Germany

${ }^{2}$ Technische Universität München, 85748 Garching, Germany

${ }^{3}$ Johannes Gutenberg-Universität Mainz, 55128 Mainz, Germany

${ }^{4}$ University of Liverpool, Liverpool, L69 7ZE, United Kingdom

${ }^{5}$ Lawrence Berkeley National Laboratory, Berkeley, California 94720-8169, USA

${ }^{6}$ University of California, Berkeley, California 94720-1460, USA

${ }^{7}$ Saha Institute of Nuclear Physics, Kolkata-700064, India

${ }^{8}$ University of Oslo, 0315 Oslo, Norway

${ }^{9}$ Lund University, 22100 Lund, Sweden

${ }^{10}$ University of Jyväskylä, 40014 Jyväskylä, Finland

${ }^{11}$ Institute of Electron Technology, 02-668 Warsaw, Poland

(Received 22 December 2009; published 21 June 2010)

The fusion-evaporation reaction ${ }^{244} \mathrm{Pu}\left({ }^{48} \mathrm{Ca}, 3-4 n\right){ }^{288,289} 114$ was studied at the new gas-filled recoil separator TASCA. Thirteen correlated decay chains were observed and assigned to the production and decay of ${ }^{288,289} 114$. At a compound nucleus excitation energy of $E^{*}=39.8-43.9 \mathrm{MeV}$, the $4 n$ evaporation channel cross section was $9.8_{-3.1}^{+3.9} \mathrm{pb}$. At $E^{*}=36.1-39.5 \mathrm{MeV}$, that of the $3 n$ evaporation channel was $8.0_{-4.5}^{+7.4} \mathrm{pb}$. In one of the $3 n$ evaporation channel decay chains, a previously unobserved $\alpha$ branch in ${ }^{281}$ Ds was observed (probability to be of random origin from background: $0.1 \%$ ). This $\alpha$ decay populated the new nucleus ${ }^{277} \mathrm{Hs}$, which decayed by spontaneous fission after a lifetime of $4.5 \mathrm{~ms}$.

Extrapolations based on the nuclear shell model performed in the 1960s led to the prediction of the existence of heavy elements in a region of the nuclear chart far away from nuclei known at the time [1]. These elements, coined "superheavy elements" (SHEs), owe their existence entirely to nuclear shell effects due to spherical proton and neutron shell closures, which stabilize them against immediate spontaneous fission (SF). SHEs were thought to be situated in a region often referred to as an "island of stability," surrounded by unstable nuclear systems.

For decades, experimental attempts using a large variety of methods were undertaken to observe SHEs. However, only in recent years have experimentalists come closer to reaching the coast of the predicted island: the discoveries of new elements with atomic numbers $Z=113-118$ were reported by collaborations working at the Flerov Laboratory of Nuclear Reactions in Dubna, Russia [2]. In 1999, the discovery of element 114 at the VASSILISSA separator using the ${ }^{242} \mathrm{Pu}\left({ }^{48} \mathrm{Ca}, 3 n\right){ }^{287} 114$ reaction was claimed [3]. Observation of the more neutron-rich ${ }^{289} 114$, produced in the ${ }^{244} \mathrm{Pu}\left({ }^{48} \mathrm{Ca}, 3 n\right)$ reaction at the Dubna GasFilled Recoil Separator (DGFRS) [4] was reported in the same year. Among the daughters, nuclides with lifetimes of tens of minutes were reported. Reports on the neighboring ${ }^{288} 114$, produced in the $4 n$ exit channel of the same nuclear fusion reaction, followed [5]. These reports were then seemingly substantiated as ${ }^{288} 114$ was also observed in an $\alpha$-decay chain from ${ }^{292} 116$ [6]. However, more recent work reported from the DGFRS, which produced additional and more detailed data, led to reassigning decay chains that were originally attributed to ${ }^{288} 114$. They are now ascribed to ${ }^{289} 114$ [7] while the original ${ }^{289} 114$ decay chain [4], which covered a time span of over $30 \mathrm{~min}$, was not reproduced. New types of decay chains were assigned to ${ }^{287} 114$ [8] as well as to ${ }^{288} 114$ [7]. The current body of data, acquired and interpreted by the Dubna group, is summarized in [2].

For a long time, all of these discovery claims remained unconfirmed, despite a number of attempts to reproduce at least part of the reported data [9-12]. Only in recent years have successful independent studies of selected reactions been reported [12-16]. DGFRS data [2] on the element 114 isotopes with masses 286 and 287 [16] were confirmed. Thus, long-standing predictions on the existence of an "island of stability" in the region of SHEs seem to have been substantiated, even though the number of observed events in every individual confirmation experiment was always less than five. Notwithstanding this limitation, it is puzzling that (i) all successful confirmation experiments reported cross sections lower than those reported from the 
TABLE I. Parameters used in the search for correlated decay chains. The rows list energy ranges and maximum lifetimes of the member denoted with the subscript number. $E$ refers to event energies in $\mathrm{MeV}$, the event type is given in parentheses. For SF, the condition for the sum of the energy deposited in the DSSSD $\left(E_{D}\right)$ and the $\operatorname{SSSSD}\left(E_{S}\right)$ is given as well as the condition for the fraction detected in the DSSSD. $\tau$ refers to lifetimes. Chains were required to start with a $3.0-15.0-\mathrm{MeV}$ evaporation residue that occurred during the beam pulse and was coincident with a MWPC signal. Decay events were required to be anticoincident with any valid MWPC signal. $\eta_{R}$ is the number of expected randomly correlated background events mimicking real decay chains in the whole experiment, and "obs" is the number of observed chains.

\begin{tabular}{|c|c|c|c|c|}
\hline & \multicolumn{2}{|c|}{ Type $1\left({ }^{289} 114\right.$ as in [2]) } & \multicolumn{2}{|c|}{ Type $2(288114$ as in [2] $)$} \\
\hline$E_{1}$ & $9.7-10.2(\alpha)$ & & $9.7-10.2(\alpha)$ & \\
\hline$\tau_{1}$ & $7 \mathrm{~s}$ & & $7 \mathrm{~s}$ & \\
\hline$E_{2}$ & $9.0-9.5(\alpha)$ & & $\left\{\begin{array}{l}E_{D}+E_{S}: \\
E_{D}:\end{array}\right.$ & $\begin{array}{l}80-400 \text { (SF) } \\
<200\end{array}$ \\
\hline $\begin{array}{l}\tau_{2} \\
E_{3} \\
\tau_{3}\end{array}$ & $\begin{array}{l}150 \mathrm{~s} \\
\left\{\begin{array}{l}E_{D}+E_{S}: \\
E_{D}:\end{array}\right. \\
50 \mathrm{~s}\end{array}$ & $\begin{array}{l}80-400(\mathrm{SF}) \\
<200\end{array}$ & $1 \mathrm{~s}$ & \\
\hline$\eta_{R}$ & 0.02 & & 0.05 & \\
\hline obs & 4 & & 9 & \\
\hline
\end{tabular}

DGFRS by factors of two or more, and (ii) unsuccessful confirmation attempts reported upper cross section limits that were below the cross sections reported from the DGFRS $[9,11,12]$. Nevertheless, the experimental cross sections are (i) unexpectedly high when compared to extrapolations of lighter systems [17], and (ii) intriguingly constant over a large range of $112 \leq Z \leq 118$. A thorough understanding of the underlying mechanism that can explain SHE production in ${ }^{48} \mathrm{Ca}$-induced reactions with actinide targets with cross sections of several picobarn is still missing. Also, the location and extension of the "island of stability" in the region of spherical SHEs are still far from being established.

In this situation, mere confirmation experiments for individual nuclides are becoming less urgent. Instead, more elaborate experiments with high statistics and, thus, with a larger discovery potential are shifting into focus. These experiments are designed to gain a better understanding of the landscape in the SHE region of the chart of nuclei as well as the SHE production mechanism. Here, we report on such an experiment using the reaction ${ }^{48} \mathrm{Ca}+$ ${ }^{244} \mathrm{Pu}$ at the newly installed Transactinide Separator and Chemistry Apparatus (TASCA) $[18,19]$, a gas-filled recoil separator with a $D Q Q$ ( $D=$ dipole, $Q=$ quadrupole $)$ magnet configuration. The design of TASCA was optimized for the study of ${ }^{48} \mathrm{Ca}$-induced fusion reactions with actinide targets. TASCA's efficiency for this nuclear reaction type is currently unsurpassed.

A pulsed (5 ms on $/ 15 \mathrm{~ms}$ off) ${ }^{48} \mathrm{Ca}^{10+}$ beam from an electron-cyclotron-resonance (ECR) ion source was accel- erated by the Universal Linear Accelerator (UNILAC) at GSI, Darmstadt, Germany, to energies of $259.4 \mathrm{MeV}$ and 254.6 MeV. The beam first passed through an induction coil to measure the beam intensity in a nondestructive way. It then passed through $(2.22 \pm 0.05)-\mu \mathrm{m}$ thick Ti target backings before entering the ${ }^{244} \mathrm{PuO}_{2}$ targets (isotopic composition: $97.9 \%{ }^{244} \mathrm{Pu} ; 1.3 \%{ }^{242} \mathrm{Pu} ; 0.7 \%{ }^{240} \mathrm{Pu}$; $<0.1 \%$ other). Three arc-shaped target segments were mounted on a target wheel, which rotated synchronously with the beam macrostructure. Over the course of the experiment, four target segments with a beam dose weighted average target thickness of $438 \mu \mathrm{g} \mathrm{cm}^{-2}{ }^{244} \mathrm{Pu}$ were irradiated with typical beam intensities of $2 \times$ $10^{12}$ particles s $^{-1}$. Initially, beam energies inside the target material were 241.3-246.2 MeV [20], corresponding to compound nucleus excitation energies, $E^{*}$, within the targets of $39.8-43.9 \mathrm{MeV}$ ( $E^{*}$ in the center of the targets, $E_{\text {c.o.t. }}^{*}=41.7 \mathrm{MeV}$ [21]; hereafter referred to as the 42$\mathrm{MeV}$ run). Later, beam energies of 236.4-241.0 MeV, resulting in $E^{*}$ within the targets of $36.1-39.5 \mathrm{MeV}$ $\left(E_{\text {c.o.t. }}^{*}=37.5 \mathrm{MeV} ; 38 \mathrm{-MeV}\right.$ run$)$, were used. Beam doses of $2.44 \times 10^{18}\left(42-\mathrm{MeV}\right.$ run) and $1.15 \times 10^{18}(38$ $\mathrm{MeV}$ run) particles were acquired. Nuclear reaction products and unreacted beam entered TASCA, which was operated in its "high transmission mode" [18]. Recoiling ions were separated by their differing magnetic rigidities, $B \cdot \rho$, in $0.8 \mathrm{mbar} \mathrm{He}$ gas inside of TASCA. The $B \cdot \rho$ of 288,289114 was estimated according to [11].

The detection system of TASCA consisted of a multiwire proportional counter (MWPC) and a focal plane detector box (FPDB). The FPDB consisted of a doublesided silicon strip detector (DSSSD) based implantation detector, with single-sided silicon strip detectors (SSSSD) mounted perpendicular in the backward hemisphere of the DSSSD to form a five-sided box configuration [22]. The DSSSD comprised 144 vertical strips with $1 \mathrm{~mm}$ pitch size on the front side and 48 horizontal strips with $1 \mathrm{~mm}$ pitch size on the back side. $90 \%$ of the total DSSSD area was active. Discrimination between ions recoiling from the target and radioactive decays of species implanted in the DSSSD was based on the (anti-)coincidence of DSSSD signals with an MWPC signal. The FPDB was calibrated with an external $\alpha$-particle source $\left({ }^{148} \mathrm{Gd},{ }^{239} \mathrm{Pu},{ }^{241} \mathrm{Am}\right.$, ${ }^{244} \mathrm{Cm}$ ) and with $\alpha$ decays from implanted transfer products $\left({ }^{214 g} \mathrm{Fr}: E_{\alpha}=8427 \mathrm{keV} ;{ }^{212 m} \mathrm{Po}: E_{\alpha}=11650 \mathrm{keV}\right)$. The energy resolution was $25 \mathrm{keV}$ full-width at half maximum (FWHM) for $8.1 \mathrm{MeV} \alpha$ particles depositing their full energy in the DSSSD (full-energy $\alpha$ particle) and $170 \mathrm{keV}$ for $\alpha$ particles that deposited a fraction of their energy inside the DSSSD and the remainder in a SSSSD (reconstructed $\alpha$ particle). The detection efficiency for an $\alpha$ particle emitted from a nucleus implanted in the active area of the DSSSD was $72 \%$, for SF it was $100 \%$. The high-energy calibration used for measuring fission fragment energies was obtained from extrapolating the calibration from the $\alpha$-energy region. The SSSSD's high- 


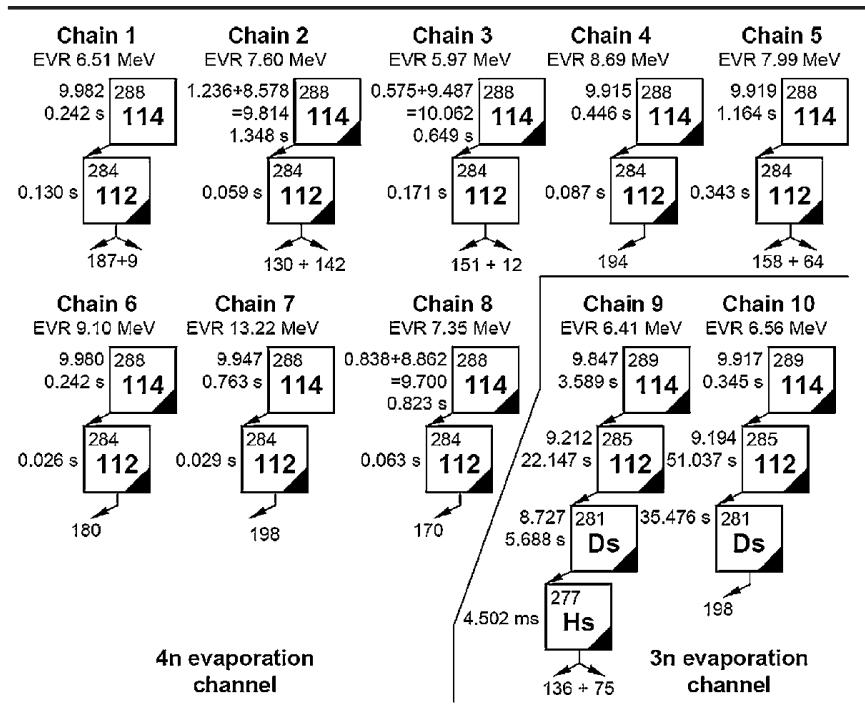

FIG. 1. Decay chains assigned to 288114 (chains 1-8) and ${ }^{289} 114$ (chains 9, 10) observed during the $42-\mathrm{MeV}$ run. Above each chain, the energy of the evaporation residue starting the chain is given. Alpha-particle energies (in $\mathrm{keV}$ ) given as $x+y=$ $z$ indicate reconstructed $\alpha$ particles that deposited $x \mathrm{keV}$ in the DSSSD and $y \mathrm{keV}$ in an SSSSD (see text). The last members of each chain decayed by SF. One arrow represents detection of a SF signal in the DSSSD, two arrows represent events where signals in both, the DSSSD and a SSSSD were registered with the numbers below giving the measured energies in $\mathrm{MeV}$. A black triangle in the lower right corner of a box indicates that the beam was off at the time of the event.

energy calibration was rather poor due to the lack of highstatistics calibration data.

The efficiency, $\varepsilon_{\text {TASCA }}$, for focusing element 114 evaporation residues (EVRs) into the DSSSD was modeled using a Monte Carlo simulation of EVR trajectories in TASCA, as described earlier $[11,23,24]$. It was $(60 \pm 6) \%$. Data acquisition was triggered by any event registering more than $300 \mathrm{keV}$ in the DSSSD or more than $500 \mathrm{keV}$ in a SSSSD. Details of the full experimental setup will be given elsewhere [25].

Guided by the results from Dubna, we searched for timeand position-correlated decay chains. The applied time and energy gates are given in Table I. Position correlations required that all members of the chain were in the same $x$ strip and in either the same $y$ strip or shared between the same two neighboring $y$ strips. Upon identification of a chain, additional EVRs $[3.0 \leq E(\mathrm{MeV}) \leq 15.0$, during beam pulse, coincident with MWPC] and $\alpha$ particles $[8.0 \leq E(\mathrm{MeV}) \leq 12.0$, anticoincident with MWPC] occurring in the same pixel as the chain, in between registration of the EVR and the terminating SF, were searched for. Two independent analysis codes were used.

Because of a damaged target segment, the background rate originating from scattered beam was increased during portions of the experiments. SFs terminating decay chains recorded during these periods were required to occur outside beam pulses. Based on the event rate, the expected number of randomly correlated background events forming chains was calculated (see Table I). Our experiment was not sensitive for the long ( $\sim 30 \mathrm{~min})$ decay chains of the type assigned to ${ }^{289} 114$ in [4] because the probability to observe random correlations over these long times was rather high.

The search yielded nine decay chains of the EVR- $\alpha$-SF type, assigned to ${ }^{288} 114$ formed in the $4 n$ evaporation channel, and four decay chains of the EVR- $\alpha-\alpha(-\alpha)$-SF type, which were assigned to ${ }^{289} 114$, as formed in the $3 n$ evaporation channel. Ten chains were measured in the 42$\mathrm{MeV}$ run and three chains in the $38-\mathrm{MeV}$ run. They are shown in Figs. 1 and 2. The agreement of our data, which is summarized in Table II, with that of [2] is good and provides an independent confirmation of this data. Only chain \#9 is different from the published data and, thus, deserves special attention. The data measured for the EVR, the first, and the second $\alpha$ particle suggest assigning the chain to ${ }^{289} 114 \rightarrow{ }^{285} 112 \rightarrow{ }^{281}$ Ds. The granddaughter, ${ }^{281} \mathrm{Ds}$ then decayed by emission of a (8.727 \pm $0.025)-\mathrm{MeV} \alpha$ particle. It was observed $5.688 \mathrm{~s}$ after the decay of ${ }^{285} 112$, during the beam-off period, where background is low. It should be noted that ${ }^{281} \mathrm{Ds}$ has undergone SF in all ten previously observed decays [2] with $T_{1 / 2}=$ $11.1_{-2.7}^{+5.0} \mathrm{~s}$. The energy region around $8.73 \mathrm{MeV}$ is near $\alpha$ lines of background activities like ${ }^{211 m, 212} \mathrm{Po}$. However, based on background rates, the probability of registering an unrelated $\alpha$-like event with properties as exhibited by the observed one is only $0.1 \%$. We therefore assign this $\alpha$ particle to a previously unobserved $\alpha$ branch in ${ }^{281}$ Ds. Considering this $\alpha$ decay and the 13 measured SF decays from [2] and our work, we arrive at an $\alpha$-decay branch $I_{\alpha}$

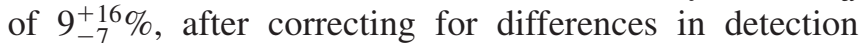
efficiency for $\alpha$ decay and SF. This results in a partial half-life for $\alpha$ decay in ${ }^{281} \mathrm{Ds}$ of $T_{1 / 2}(\alpha)=140_{-90}^{+510} \mathrm{~s}$. This is consistent with the formalism linking the $\alpha$-particle energy, $E_{\alpha}$, and $T_{1 / 2}(\alpha)$ in [26], which indicates that an unhindered $\alpha$ decay with $E_{\alpha}=8.73 \mathrm{MeV}$ should have $T_{1 / 2} \sim 370 \mathrm{~s}$. The chain was then terminated $4.5 \mathrm{~ms}$ later by $\mathrm{SF}$ of the $\alpha$-decay daughter of ${ }^{281} \mathrm{Ds}$, i.e., the new nucleus ${ }^{277} \mathrm{Hs}$ with $Z=108$ and $N=169$. In addition to the members shown in Fig. 1, one further EVR with an energy of $3.73 \mathrm{MeV}$ was registered inside that chain $19.416 \mathrm{~s}$ after the EVR starting the chain. Based on the rate of EVR-like events, the probability for finding an additional, unrelated EVR within chain \#9 was evaluated to be $9.2 \%$. Hence, this event is considered to be unrelated to chain $\# 9$ as given in Fig. 1. There was only one additional EVR observed within any of the other chains, which is compatible with expectations based on our event rate.

$Z=108$ has been shown to be a deformed proton shell closure in lighter isotopes with $N \sim 162$ [27]. The observed lifetime of ${ }^{277} \mathrm{Hs}$ is short compared to half-lives of the lighter $\mathrm{Hs}$ isotopes, which are situated near the deformed neutron shell closure at $N=162$. SF has never been observed in Hs isotopes with $N>156$ and a conser- 


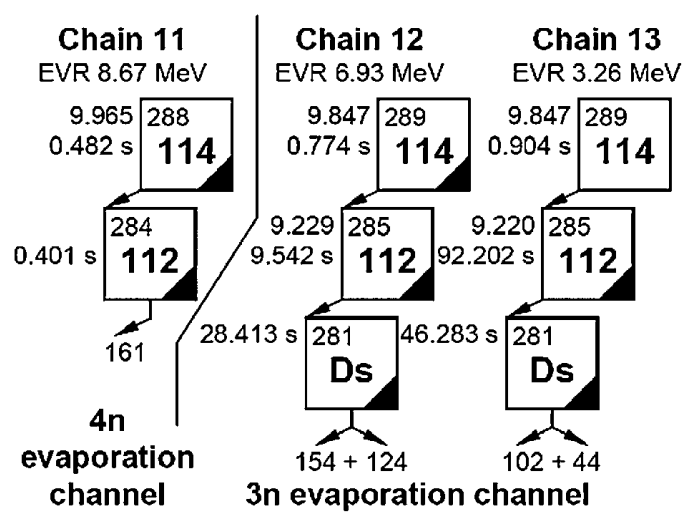

FIG. 2. Same as Fig. 1, but showing decay chains assigned to ${ }^{288} 114$ (chain 11) and ${ }^{289} 114$ (chains 12 and 13) observed during the $38-\mathrm{MeV}$ run.

vative lower limit on $T_{1 / 2}(\mathrm{SF})$ for the isotopes around $N=$ $162,{ }^{269-271} \mathrm{Hs}$, is of the order of $10 \mathrm{~s}$ [27,28]. The 4.5-ms SF lifetime observed for ${ }^{277} \mathrm{Hs}$ is much shorter, indicating a weakening of shell stabilization in the $N=169$ nucleus ${ }^{277}$ Hs.

For the even $\mathrm{Hs}$ isotopes neighboring ${ }^{277} \mathrm{Hs}$, macroscopic-microscopic model calculations [29] predict $T_{1 / 2}(\mathrm{SF})$ of $46 \mathrm{~ms}\left({ }^{276} \mathrm{Hs}\right)$ and $0.98 \mathrm{~ms}\left({ }^{278} \mathrm{Hs}\right)$. The geometric mean of the predicted half-lives of neighboring ${ }^{276,278} \mathrm{Hs}$ is $6.7 \mathrm{~ms}$, which is similar to our observed lifetime. The odd neutron is expected to hinder SF decay by several orders of magnitude. Thus, our measured 4.5-ms lifetime indicates that the drop in $T_{1 / 2}(\mathrm{SF})$ when increasing $N$ above 162 may be more severe than suggested by [29]. This is in line with measured $T_{1 / 2}$ of SF-decaying even element 112 isotopes in this region of $N$, where experimental values are smaller than theoretical ones by 1 to 2 orders of magnitude.

It is interesting to note that ${ }^{275} \mathrm{Hs}(N=167)$ decays by $\alpha$-particle emission with $T_{1 / 2}=0.19$ s [2]. The experimental trend with prevalent $\alpha$ decay in Hs isotopes with $N \sim 157-167$, but predominant SF in lighter as well as in more neutron-rich isotopes is close to the calculated one [29], which suggests $\alpha$ decay from $N=154$ to $N=166$ but $\mathrm{SF}$ for $N>168$ to be the dominant decay mode. This strongly indicates that stability vanishes with increasing distance from $N=162$. In line with this, and close to the theoretical results of [29], is the observation of a shift in dominant decay mode from $\alpha$ decay in the $N=169 \mathrm{nu}-$ cleus ${ }^{279}$ Ds [2] toward SF in $N=171{ }^{281}$ Ds.

The $B \cdot \rho$ of element 114 EVRs in 0.8 mbar He was measured to $(2.29 \pm 0.11) \mathrm{T} \mathrm{m}$ in the $42-\mathrm{MeV}$ run, where the best statistics was obtained.

The measured cross sections at $E^{*}=36.1-39.5 \mathrm{MeV}$ were $8.0_{-4.5}^{+7.4} \mathrm{pb}$ for the $3 n$ channel and $2.8_{-2.1}^{+4.2} \mathrm{pb}$ for the $4 n$ channel. At $E^{*}=39.8-43.9 \mathrm{MeV}$, cross sections were $3.5_{-2.0}^{+3.3} \mathrm{pb}\left(3 n\right.$ channel) and $9.8_{-3.1}^{+3.9} \mathrm{pb}(4 n$ channel $)$. Error bars include statistical uncertainties at the $68.3 \%$ confidence level and are calculated according to [30]. The systematic uncertainty of the cross section data is estimated to be $14 \%$. In contrast to any other experiment performed outside of the DGFRS collaboration, we are able to fully confirm the large cross sections reported from the DGFRS for superheavy element production. In fact, our measured cross sections for the $3 n$ and $4 n$ evaporation channels are higher than those reported from the DGFRS. They conform to the excitation function shape trends from the DGFRS [2].

In summary, the nuclear fusion reaction ${ }^{244} \mathrm{Pu}\left({ }^{48} \mathrm{Ca}, 3-4 n\right)$ was studied at the new gas-filled recoil separator TASCA. Thirteen decay chains were observed and assigned to ${ }^{288,289} 114$. Cross sections, decay modes, $\alpha$-particle energies, and half-lives agree with those reported from the DGFRS Collaboration [2]. The combination of our results and those of [2] leads to more precise decay data (see Table II). Moreover, we have discovered a previously unobserved $\alpha$-decay branch in ${ }^{281}$ Ds with $E_{\alpha}=$ $(8.727 \pm 0.025) \mathrm{MeV}$ and one decay of the new nucleus ${ }^{277} \mathrm{Hs}$, which decayed by SF after $4.5 \mathrm{~ms}$. These observations are close to results of calculations based on the macroscopic-microscopic model [29] and yield important information on the competition between $\alpha$ decay and SF in the region of $N \sim 170$ isotones around $Z=108$, which is a deformed proton shell closure near $N=162$. In contrast to results of independent confirmation experiments with rather low statistics $[14,16]$, our measured cross sections of $9.8_{-3.1}^{+3.9} \mathrm{pb}$ for the $4 n$ evaporation channel and of $8.0_{-4.5}^{+7.4} \mathrm{pb}$ for the $3 n$ evaporation channel are even higher than, but still in agreement with, those reported from the

TABLE II. Decay properties of isotopes observed in this work. Alpha-particle energies, $E_{\alpha}$, are based solely on "full-energy $\alpha$ particles" (see text).

\begin{tabular}{lclccc}
\hline \hline Isotope & $\begin{array}{c}\text { Decay mode } \\
\text { this work and [2] }\end{array}$ & $\begin{array}{l}E_{\alpha}(\mathrm{MeV}) \\
\text { this work }\end{array}$ & this work & $\begin{array}{c}\text { Half-life } \\
\text { Lit. [2] }\end{array}$ & combined \\
\hline${ }^{289} 114$ & $\alpha$ & $9.87 \pm 0.03$ & $0.97_{-0.32}^{+0.97} \mathrm{~s}$ & $2.6_{-0.7}^{+1.2} \mathrm{~s}$ & $2.1_{-0.4}^{+0.8} \mathrm{~s}$ \\
${ }^{288} 114$ & $\alpha$ & $9.95 \pm 0.03$ & $0.47_{-0.12}^{+0.24} \mathrm{~s}$ & $0.80_{-0.16}^{+0.27} \mathrm{~s}$ & $0.69_{-0.11}^{+0.17} \mathrm{~s}$ \\
${ }^{285} 112$ & $\alpha$ & $9.21 \pm 0.03$ & $30_{-10}^{+30} \mathrm{~s}$ & $29_{-7}^{+13} \mathrm{~s}$ & $29_{-6}^{+11} \mathrm{~s}$ \\
${ }^{284} 112$ & $\mathrm{SF}$ & & $101_{-25}^{+50} \mathrm{~ms}$ & $97_{-19}^{+13} \mathrm{~ms}$ & $99_{-16}^{+24} \mathrm{~ms}$ \\
${ }^{281} \mathrm{Ds}$ & $\mathrm{SF}\left(91_{-16}^{+7} \%\right) / \alpha\left(9_{-7}^{+16} \%\right)$ & $8.73 \pm 0.03$ & $20_{-7}^{+20} \mathrm{~s}$ & $11.1_{-2.7}^{+50} \mathrm{~s}$ & $13_{-3}^{+5} \mathrm{~s}$ \\
${ }^{277} \mathrm{Hs}$ & $\mathrm{SF}$ & & $3_{-1}^{+15} \mathrm{~ms}$ & $($ Not observed) & $3_{-1}^{+15} \mathrm{~ms}$ \\
\hline \hline
\end{tabular}


DGFRS. These high cross sections call for investigations of the details of the production mechanism. Production rates that follow from these values encourage using this nuclear reaction to produce relatively long-lived isotopes of element 114, in particular, for envisaged chemical investigations of this superheavy element or for $\gamma$-spectroscopic studies that may help to shed light on the underlying nuclear structure in this region of the heaviest known nuclei.

We thank the ECR ion source and UNILAC staff for providing excellent and stable ${ }^{48} \mathrm{Ca}$ beams. $\mathrm{H}$. Brand and the GSI Experimental Electronics department, $\mathrm{H}$. Grösslhuber, G. Matheis, and R. Bühnemann from the machine shop at the institute of radiochemistry, TU Munich, as well as V. Gorshkov provided technical support. L. Stavsetra provided preliminary BGS results for the ${ }^{48} \mathrm{Ca}+{ }^{242} \mathrm{Pu}$ reaction prior to publication, which we gratefully acknowledge. This work was financially supported by the German BMBF (06MT247I, 06MT248, 06MZ223I); the GSI-F\&E (MT/TÜR, MZJVKR); the Swedish Science Council; the U.S. D.O.E. under Contract No. DE-AC0205CH11231 and the NNSA under Contract No. DE-FC5208NA28752; the Norwegian Research Council (Project No. 177538); the government of India-XIth five year plan project TADDS.

*c.e.duellmann@gsi.de

Present address: Paul Scherrer Institute, 5232 Villigen, Switzerland.

[1] W. D. Myers and W. J. Swiatecki, Nucl. Phys. 81, 1 (1966).

[2] Yu. Oganessian, J. Phys. G 34, R165 (2007).

[3] Yu. Ts. Oganessian et al., Nature (London) 400, 242 (1999).
[4] Yu.Ts. Oganessian et al., Phys. Rev. Lett. 83, 3154 (1999).

[5] Yu. Ts. Oganessian et al., Phys. Rev. C 62, 041604(R) (2000).

[6] Yu. Ts. Oganessian et al., Phys. Rev. C 63, 011301(R) (2000).

[7] Yu. Ts. Oganessian et al., Phys. Rev. C 69, 054607 (2004).

[8] Yu. Ts. Oganessian et al., Phys. Rev. C 70, 064609 (2004).

[9] R. Eichler et al., Radiochim. Acta 94, 181 (2006).

[10] W. Loveland et al., Phys. Rev. C 66, 044617 (2002).

[11] K. E. Gregorich et al., Phys. Rev. C 72, 014605 (2005).

[12] R. Eichler et al., Nucl. Phys. A787, 373 (2007).

[13] R. Eichler et al., Angew. Chem., Int. Ed. 47, 3262 (2008).

[14] S. Hofmann et al., Eur. Phys. J. A 32, 251 (2007).

[15] R. Eichler et al., Nature (London) 447, 72 (2007).

[16] L. Stavsetra et al., Phys. Rev. Lett. 103, 132502 (2009).

[17] S. Hofmann, Lect. Notes Phys. 764, 203 (2009).

[18] A. Semchenkov et al., Nucl. Instrum. Methods Phys. Res., Sect. B 266, 4153 (2008).

[19] M. Schädel, Eur. Phys. J. D 45, 67 (2007).

[20] J. F. Ziegler, Nucl. Instrum. Methods Phys. Res., Sect. B 219-220, 1027 (2004).

[21] W. D. Myers and W. J. Świątecki, Nucl. Phys. A601, 141 (1996).

[22] A. Gorshkov et al. (to be published).

[23] K. E. Gregorich et al., Eur. Phys. J. A 18, 633 (2003).

[24] K.E. Gregorich et al., Gesellschaft für Schwerionenforschung mbH, Darmstadt, Germany, Report No. 20071, 2007.

[25] J. M. Gates et al. (to be published).

[26] A. Parkhomenko and A. Sobiczewski, Acta Phys. Pol. B 36, 3095 (2005).

[27] J. Dvorak et al., Phys. Rev. Lett. 97, 242501 (2006).

[28] J. Dvorak et al., Phys. Rev. Lett. 100, 132503 (2008).

[29] R. Smolańczuk, J. Skalski, and A. Sobiczewski, Phys. Rev. C 52, 1871 (1995).

[30] W. Brüchle, Radiochim. Acta 91, 71 (2003). 